\title{
$\pi$ ingenierías
}

\section{Estimación del carbono almacenado en la biomasa aérea de un bosque húmedo tropical en Paimadó, Chocó}

\section{Estimated carbon content in the aerial biomass of a tropical rainforest in Paimado, Choco}

\author{
Nathaly Aseneth Paipa Rios \\ Universidad Distrital Francisco José de Caldas \\ nathalypaipa@gmail.com
}

\author{
Max Alejandro Triana Gómez \\ Universidad Distrital Francisco José de Caldas \\ maxtriana2@gmail.com
}

(Tipo de Artículo: Investigación científica y tecnológica. Recibido: 04/08/2017. Aprobado: 19/09/2017)

\begin{abstract}
Resumen. En Paimadó cabecera municipal de Río Quito (Chocó) se estima el carbono contenido en la biomasa aérea de un bosque perteneciente al zonobioma húmedo tropical. Para el cálculo de la biomasa se utilizan trece modelos alométricos reportados como los que mejor se ajustan al ecosistema según literatura especializada, estos son aplicados a las diez especies con mayor peso ecológico dentro del bosque, por representar cerca del $60 \%$ de la biomasa total de las especies encontradas. El inventario forestal consiste en un muestreo bietápico en bloques y fajas en 250 parcelas circulares de 0.05 ha seleccionadas al azar, se miden las variables dasométricas de altura total y diámetro a la altura del pecho, y para el análisis estadístico se utiliza el programa computacional de licencia libre R Project. El carbono almacenado se determina con base en la fracción propuesta por el IPCC y corresponde al $50 \%$ de la biomasa total. Los resultados determinan que el bosque húmedo tropical en Paimadó contiene un promedio de 78.49 t de biomasa y 39.24 t de carbono por cada 0.05 ha.
\end{abstract}

Abstract. In Paimado municipal head of Rio Quito (Choco) the carbon contained in the aerial biomass of a forest belonging to a tropical humid zonobioma was evaluated. For the calculate of the biomass, thirteen allometric models were used reported as the best to fit the ecosystem according to specialized literature, these were applied to the 10 species with greater ecological weight within the forest, since they represent about the $60 \%$ of the total biomass of the species found. The forest inventory consisted in a twostage sampling in blocks and girdles in 250 circular plots of 0.05 ha randomly selected, the height and diameter at breast height variables were measured, for the statistical analysis the free software $R$ Project was used. The stored carbon was determined based on the fraction proposed by the IPCC and corresponds to $50 \%$ of the total biomass. The results determined that the tropical rainforest in Paimado contains 78.49 t of biomass and 39.24 t of carbon for each 0.05 ha.

Palabras clave. Biomasa; bosque húmedo tropical; carbono almacenado; inventario; modelos alométricos.

Keywords. Allometric models; biomass; carbon stored; tropical rainforest; stock.

DOI: $10.21500 / 20275846.3180$

\section{Introducción}

El dióxido de carbono $\left(\mathrm{CO}_{2}\right)$ atmosférico es incorporado a los procesos metabólicos del bosque gracias a la fotosíntesis, donde tiene lugar la generación de materias primas como la glucosa, permitiendo la aparición del follaje, ramas y demás estructuras necesarias para el desarrollo del árbol [1]. Una vez que los bosques fijan en sus estructuras vivas el dióxido de carbono atmosférico, lo acumulan en su biomasa y parte lo transfieren al mantillo y al suelo por descomposición, constituyendo las reservas de carbono [2]. La composición florística, la edad y la abundancia de población de cada estrato por comunidad vegetal son los principales factores que influyen en la capacidad que tienen los ecosistemas forestales para almacenar carbono en forma de biomasa aérea [3], una estimación indirecta del material vegetal permite inferir dicha cantidad de biomasa a partir de relaciones alométricas que toman como base el tamaño y dimensiones de los árboles; siendo este el método más práctico que se puede emplear [4].

La constante dinámica propia de los bosques tropicales, sumado a su extensa área, hace que tengan un papel vital en la regulación de la cantidad de dióxido de carbono en la atmósfera $[5,6]$, por lo que conocer el ciclo del carbono y su dinámica en el bosque a través del cálculo de sumideros, es primordial para plantear estrategias viables que contribuyan a alcanzar compromisos de mitigación de la variabilidad climática [7].

Las estimaciones de biomasa en los bosques de Colombia se realizan en su mayoría para la región Andina y Amazónica. Sin embargo, en la referencia [8] se presentan algunos datos para la región Pacífica. El 
total de carbono almacenado en los bosques colombianos estuvo por encima de los 7800 millones de toneladas para el año 2007, con mayores contenidos promedio de reserva en el bosque muy húmedo tropical (135.29 t/ha) y el bosque húmedo tropical (131.87 t/ha). El Pacífico ocupa el segundo lugar después de la Amazonía, como la región con los promedios más altos de carbono (131 t/ha), aun cuando su extensión total es de las menores con siete millones de hectáreas. Tendencia que se repite en el área de estudio; el departamento del Chocó, en donde se estimó que su contenido promedio de carbono está por encima de las $135 \mathrm{t} / \mathrm{ha}$, ocupando el tercer lugar a nivel departamental.

Los bosques del municipio de Río Quito son fuertemente perturbados debido a la histórica extracción minera específicamente de oro y platino [9], desencadenando procesos de deforestación y fragmentación que influyen en la acumulación de carbono. Por tal razón, en el presente estudio se propone estimar la biomasa aérea y el carbono almacenado en un bosque perteneciente al zonobioma húmedo tropical de la cuenca baja del Río Quito, determinando las diez especies con mayor Índice de Valor de Importancia, comprobando la existencia o ausencia de diferencias significativas entre estas en relación a los modelos alométricos y estableciendo finalmente cuál es la que mayor carbono almacena.

\section{Metodología}

\section{1 Área de estudio y caracterización}

El estudio tiene lugar en Paimadó, municipio de Río Quito, departamento del Chocó, Pacífico colombiano; ubicado en la subcuenca baja del río Quito, margen izquierda del río Atrato, entre los $5^{\circ} 25^{\prime} 00^{\prime \prime}$ de latitud Norte y los $76^{\circ} 40^{\prime} 00^{\prime \prime}$ de longitud Oeste. La cabecera municipal Paimadó, está ubicada a más de tres horas desde la ciudad de Quibdó accediendo por el cauce del Río Quito, alternada con acceso vial por la panamericana Las Animas-Nuquí por un ramal de 8.5 $\mathrm{km}$.

Río Quito tiene una altura promedio de 125 m.s.n.m. y una extensión de $700 \mathrm{~km}^{2}$, limitando al norte con la capital del departamento Quibdó, al sur con Cantón de San Pablo y Certegui, al oriente con Atrato y al Occidente con Alto Baudó. Lo conforman ocho corregimientos, entre ellos Paimadó, su cabecera municipal; presenta una temperatura media anual de $25^{\circ} \mathrm{C}$ y valores de precipitación entre los $6750 \mathrm{~mm}$ y $7750 \mathrm{~mm}$ anuales [11].
El bioma por excelencia es el bosque húmedo tropical, con bosques naturales y vegetación secundaria en el zonobioma húmedo tropical Pacífico - Atrato [10], en general el bosque tiene un aproximado de 250 especies por hectárea [11].

El aprovechamiento forestal es el pilar económico del municipio, sin embargo, no se hace intervención planificada y no se cuenta con programas de reforestación, por lo que el bosque está sometido a una fuerte presión por el incremento constante en los volúmenes de explotación. La actividad minera que tiene lugar aguas arriba de Paimadó, altera de igual forma el paisaje, dejando pérdidas de vegetación en más de 19 hectáreas de bosque natural en los meandros existentes entre la desembocadura de la quebrada Manuel Marín y $1.5 \mathrm{~km}$ aguas arriba de la desembocadura de la quebrada Chigorodó [9].

\subsection{Toma de datos}

El muestro es de tipo bietápico organizado en bloques y fajas, con una probabilidad del $95 \%$ y una intensidad de $0.08 \%$, se establece al azar 250 parcelas circulares de 0.05 ha cada una en un bosque natural perteneciente al zonobioma húmedo tropical Pacífico - Atrato; evaluando aspectos de la composición florística y estructura de la vegetación descritos en la metodología propuesta en [12].

Se tienen en cuenta las variables dasométricas típicas de un inventario forestal, iniciando con la selección de individuos de más de $10 \mathrm{~cm}$ de diámetro a la altura del pecho (DAP medido a $1.30 \mathrm{~m}$ de altura), luego la toma de altura total y comercial en metros. Finalmente, la identificación de las especies; cuando no se reconoce el individuo, se recolecta material vegetal para su posterior identificación taxonómica.

\subsection{Análisis de datos}

Se transcribe, digitaliza, filtra y depura la información registrada en las planillas y se inicia con el cálculo del Índice de Valor de Importancia (IVI) tomando en cuenta las variables de abundancia, frecuencia y dominancia, para definir cuáles especies contribuyen en mayor medida al carácter y estructura del bosque [13], asignando a cada una su categoría de importancia dentro del muestreo $[14,15]$. Las diez especies con mayor IVI son seleccionadas para aplicarles trece modelos alométricos de estimación indirecta de biomasa descritos en la Tabla 1, desarrollados por diferentes autores. 
Tabla 1. Modelos alométricos utilizados para estimar la biomasa aérea en zonobioma húmedo tropical

\begin{tabular}{|c|c|c|}
\hline Modelo & Descripción & Autor \\
\hline 3 & $\mathrm{~B}=\mathrm{EXP}\left(-1.663+\left(2.37^{*} \mathrm{LN}(\mathrm{DAP})\right)\right)$ & Álvarez, 2011 \\
\hline 6 & $\mathrm{~B}=\mathrm{EXP}\left(-2,289+\left(0,932^{*} \mathrm{LN}\left((\mathrm{DAP})^{\wedge} 2^{*}(\text { (ALTURA metros })^{*} 0,65\right)\right)\right)$ & Álvarez, 2011 \\
\hline 7 & $\mathrm{~B}=\mathrm{EXP}\left(-2.19+\left(2.54^{*} \mathrm{LN}(\mathrm{DAP})\right)\right)$ & Chave, 2007 \\
\hline 8 & $\mathrm{~B}=\mathrm{EXP}[-2.289+2.649 * \ln (\mathrm{DAP})-0.021 * \ln (\mathrm{DAP} 2)]$ & IPCC, 2005 \\
\hline 11 & $\mathrm{~B}=\mathrm{EXP}\left(-2.904+\left(0.993^{*} \mathrm{LN}\left(0.65^{*}(\mathrm{~B})^{\wedge} 2^{*}(\mathrm{ALTURA} \text { metros })^{*}\right)\right)\right)$ & Overman, 1990 \\
\hline 12 & $\mathrm{~B}=\mathrm{EXP}\left(-2.977+\mathrm{LN}\left(0.65^{*}(\mathrm{DAP})^{\wedge} 2^{*}(\mathrm{ALTURA}\right.\right.$ metros $\left.\left.)\right)\right)$ & Chave, 2005 \\
\hline 13 & $\mathrm{~B}=\operatorname{EXP}\left(-2.187+\left(0.916^{*} \mathrm{LN}\left(0.65^{\star}(\mathrm{DAP})^{\wedge} 2^{\star}(\mathrm{ALTURA}\right.\right.\right.$ metros $\left.\left.\left.)\right)\right)\right)$ & Chave, 2005 \\
\hline
\end{tabular}

Luego del procesamiento, los datos son ingresados al programa computacional de licencia libre $R$ Project versión 3.1.1., para su análisis estadístico descriptivo: media, desviación estándar, intervalos de confianza y normalidad de datos mediante prueba Shapiro-Wilk, e inferencial: pruebas de varianza Kruskal-Wallis, para evaluar las diferencias significativas de las variables estructurales y la biomasa por especie y por clase diamétrica. En cuanto al cálculo del carbono almacenado, se toma como el $50 \%$ de la biomasa estimada de cada modelo seleccionado para especie y para el zonobioma en general según lo estipulado en [16].

\section{Resultados}

\subsection{Indice de valor de importancia y selección de especies}

La Tabla 2 resume las diez especies con mayor IVI en el zonobioma húmedo tropical estudiado, se muestran los valores de abundancia relativa que reflejan la relación porcentual del número de individuos de la especie con respecto al total de individuos de la parcela; valores de frecuencia siendo la probabilidad de encontrar la especie en la muestra; finalizando con el valor de dominancia que representa la importancia de la especie en función de su desarrollo o biomasa.

Tabla 2. Especies con mayor Índice de valor de importancia en el zonobioma húmedo tropical

\begin{tabular}{|c|c|c|c|c|c|c|}
\hline Nombre científico & Familia & $\begin{array}{l}\text { Abundancia } \\
\text { relativa }\end{array}$ & $\begin{array}{l}\text { Frecuencia } \\
\text { relativa }\end{array}$ & $\begin{array}{l}\text { Dominancia } \\
\text { relativa }\end{array}$ & IVI & IVI\% \\
\hline Aspidosperma sp. & Apocynaceae & 5.10 & 1.34 & 12.88 & 19.33 & 6.44 \\
\hline Eschweilera coriacea (DC.) S.A.Mori & Lecythidaceae & 5.57 & 1.34 & 5.83 & 12.74 & 4.24 \\
\hline Dacryodes colombiana Cuatrec. & Burseraceae & 6.36 & 1.34 & 4.48 & 12.18 & 4.06 \\
\hline Iryanthera juruensis Warb. & Myristicaceae & 3.38 & 1.34 & 3.21 & 7.94 & 2.64 \\
\hline Inga acrocephala Steud. & Leguminosae & 3.43 & 1.34 & 2.35 & 7.13 & 2.37 \\
\hline Oenocarpus bataua Mart. & Arecaceae & 3.48 & 1.34 & 1.02 & 5.85 & 1.95 \\
\hline Pentaclethra macroloba (Willd.) Kuntze & Leguminosae & 1.67 & 1.34 & 2.83 & 5.84 & 1.94 \\
\hline
\end{tabular}

Para simplificar el cálculo y análisis del carbono almacenado en el zonobioma húmedo tropical, se selecciona el Índice de valor de importancia (IVI) por tratarse de un buen descriptor del peso ecológico de las especies dentro del ecosistema analizado; las diez especies seleccionadas representan el $33 \%$ de ese valor. Por lo tanto, constituyen una buena aproximación para inferir la cantidad de biomasa aérea que contiene el bosque, además permiten una comparación directa y clara con los resultados arrojados por la totalidad del zonobioma, y con otros estudios similares.
La familia Leguminosae tiene la mayor representación con tres especies en el listado ( $A$. jupunba, I. acrocephala y $P$. macroloba) con un $7.01 \%$ de IVI del $100 \%$ total. Sin embargo, Aspidosperma sp. de la familia Apocynaceae es la que mayor IVI reporta con un $6.4 \%$ siendo la especie con la dominancia más alta, es decir la de mayor importancia en función de su desarrollo o biomasa. La especie más abundante en todo el zonobioma es Dacryodes colombiana, Pentaclethra macroloba es la menos abundante y por su parte Oenocarpus bataua es la menos dominante en términos de ocupación, consecuente por tratarse de una palma. 
Es destacable que estas especies corresponden al estado maduro de la sucesión, y que la presencia de palmas obedece a una dinámica de perturbaciones típica de estos bosques.

\subsection{Modelos alométricos y cálculo de biomasa}

En la Tabla 3 se muestra el compilado de los coeficientes de correlación de Spearman calculados para los trece modelos, todos contemplaron el diámetro a la altura del pecho en su ecuación, los modelos 5,6 , 11,12 y 13 incluyeron la variable dasométrica de altura total.

Tabla 3. Coeficientes de correlación de Spearman de trece modelos alométricos

\begin{tabular}{lllllllllll}
\hline Mod & $\begin{array}{l}\text { Aspidosperma } \\
\text { sp. }\end{array}$ & $\begin{array}{l}\text { Micropholis } \\
\text { guyanensis }\end{array}$ & $\begin{array}{l}\text { Eschweilera } \\
\text { coriacea }\end{array}$ & $\begin{array}{l}\text { Dacryodes } \\
\text { colombiana }\end{array}$ & $\begin{array}{l}\text { Abarema } \\
\text { jupunba }\end{array}$ & $\begin{array}{l}\text { Iryanthera } \\
\text { juruensis }\end{array}$ & $\begin{array}{l}\text { Inga } \\
\text { acrocephala }\end{array}$ & $\begin{array}{l}\text { Oenocarpus } \\
\text { bataua }\end{array}$ & $\begin{array}{l}\text { Pentaclethra } \\
\text { macroloba }\end{array}$ & $\begin{array}{l}\text { Guarea } \\
\text { glabra }\end{array}$ \\
\hline 1 & 0.992 & 0.997 & 0.993 & 0.994 & 0.993 & 0.993 & 0.991 & 0.971 & 0.997 & 0.993 \\
\hline 2 & 0.992 & 0.997 & 0.993 & 0.994 & 0.993 & 0.993 & 0.991 & 0.971 & 0.997 & 0.993 \\
\hline 3 & 0.992 & 0.997 & 0.993 & 0.994 & 0.993 & 0.993 & 0.991 & 0.971 & 0.997 & 0.993 \\
\hline 4 & 0.992 & 0.997 & 0.993 & 0.994 & 0.993 & 0.993 & 0.991 & 0.971 & 0.997 & 0.974 \\
\hline 5 & 0.985 & 0.990 & 0.985 & 0.987 & 0.983 & 0.981 & 0.980 & 0.989 \\
\hline 6 & 0.985 & 0.990 & 0.985 & 0.987 & 0.983 & 0.981 & 0.980 & 0.885 & 0.974 & 0.989 \\
\hline 7 & 0.992 & 0.997 & 0.993 & 0.994 & 0.993 & 0.993 & 0.991 & 0.971 & 0.997 & 0.993 \\
\hline 8 & 0.992 & 0.997 & 0.993 & 0.994 & 0.993 & 0.993 & 0.991 & 0.971 & 0.997 & 0.993 \\
\hline 9 & 0.992 & 0.997 & 0.993 & 0.994 & 0.993 & 0.993 & 0.991 & 0.971 & 0.997 \\
\hline 10 & 0.992 & 0.997 & 0.993 & 0.994 & 0.993 & 0.993 & 0.991 & 0.971 & 0.997 & 0.993 \\
\hline 11 & 0.985 & 0.990 & 0.985 & 0.987 & 0.983 & 0.981 & 0.980 & 0.885 & 0.974 \\
\hline 12 & 0.985 & 0.990 & 0.985 & 0.987 & 0.983 & 0.981 & 0.980 & 0.885 & 0.974 \\
\hline 13 & 0.985 & 0.990 & 0.985 & 0.987 & 0.983 & 0.981 & 0.980 & 0.885 & 0.974 \\
\hline
\end{tabular}

Los coeficientes de correlación de Spearman muestran la relación lineal que tienen las variables muestreadas, la asociación resulta ser positiva en todos los modelos y para todas las especies. La menor correlación a nivel de especies la reporta Oenocarpus bataua (0.885), comportamiento que se repite al comparar los modelos. Sin embargo, este resultado debe tomarse con cautela, porque se trata de una especie no maderable y posee una relación de esbeltez diferente a los arboles; la mayor correlación en promedio es para las especies Micropholis guyanensis, seguida de Guarea glabra.

Se observa que los valores resultaron muy cercanos entre sí y el modelo que mejor se ajusta al zonobioma húmedo tropical para todas las especies es el modelo 9.

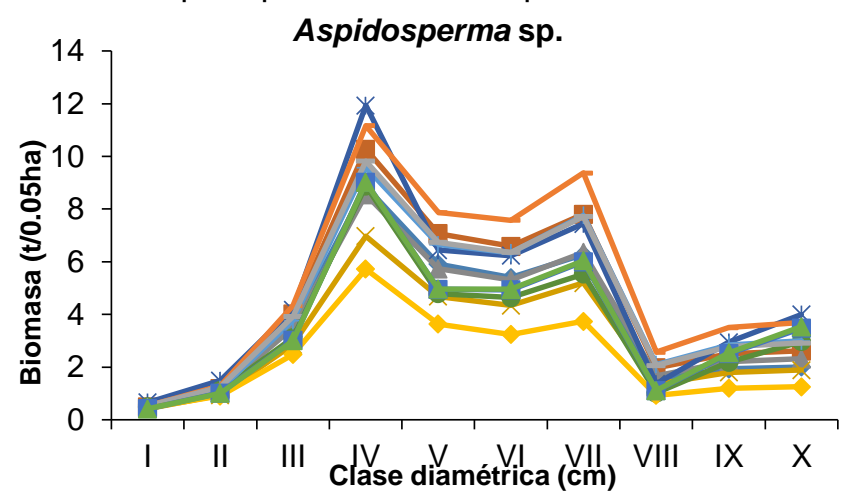

Este modelo contempla únicamente la variable dasómetrica de diámetro a la altura del pecho. Además, los modelos $5,6,11,12$ y 13 que incluyen la variable altura total reportan los menores coeficientes de correlación.

Los modelos alométricos calculan la cantidad de biomasa por especie y para la totalidad del ecosistema muestreado, los valores se observan en la Figura 1, donde se relaciona la cantidad de biomasa en toneladas por cada 0.05 ha para clases diamétricas agrupadas cada $10 \mathrm{~cm}$ de diámetro a la altura del pecho $(I=10-20$ $\mathrm{cm}, I I=20-30 \mathrm{~cm}, I I I=30-40 \mathrm{~cm}, I V=40-50 \mathrm{~cm}, \mathrm{VI}=50-60$ $\mathrm{cm}, V I I=60-70 \mathrm{~cm}, V I I I=70-80 \mathrm{~cm}, I X=80-90 \mathrm{~cm}, X=90-$ $100 \mathrm{~cm}, X \mathrm{I}=100-110 \mathrm{~cm}$ ), según la especie.

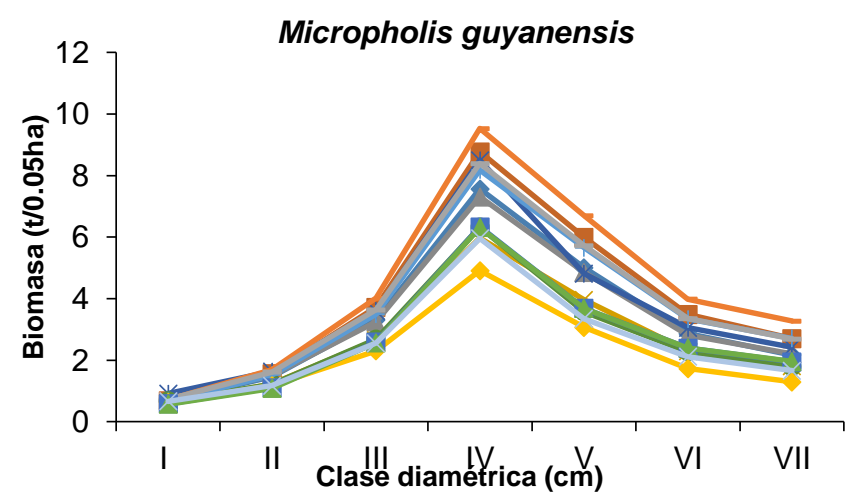


Figura 1. Distribución de biomasa aérea por clase diamétrica para especies y zonobioma según modelos alométricos $(1 / 3)$
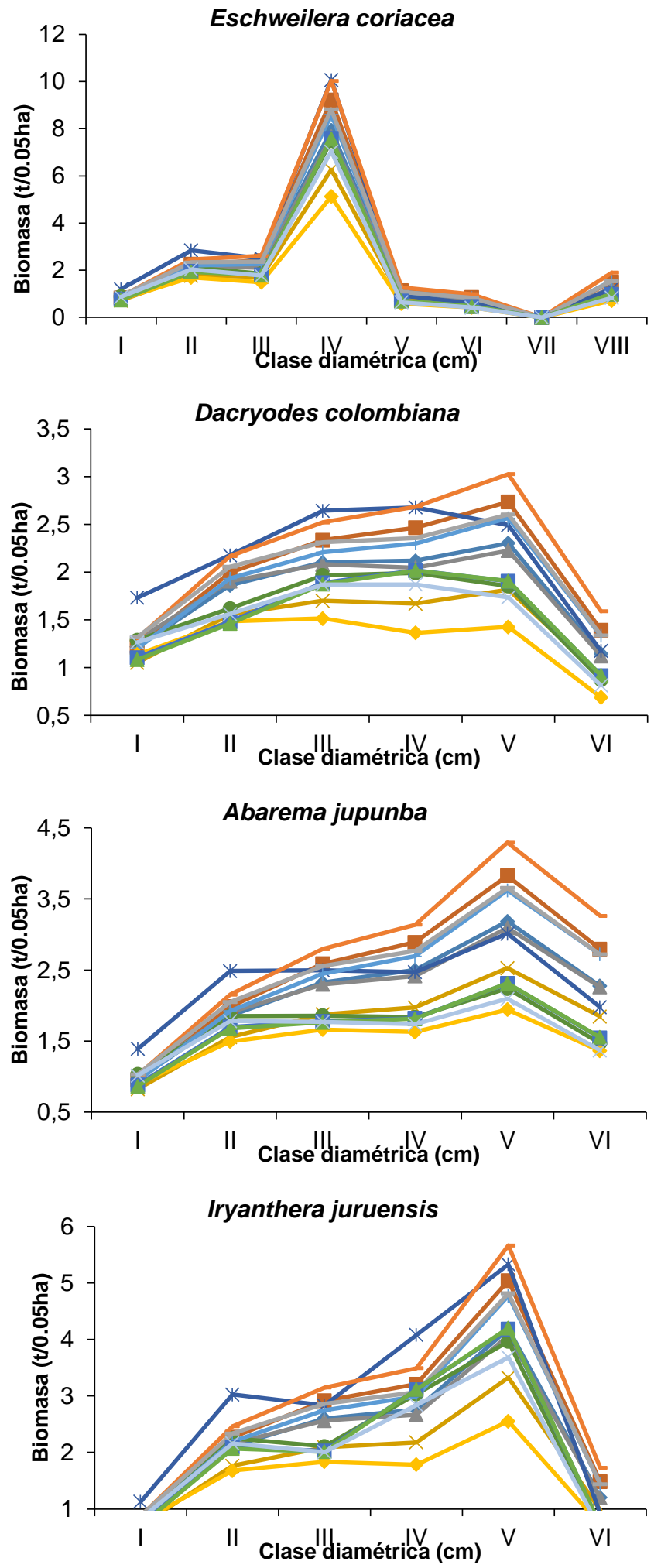
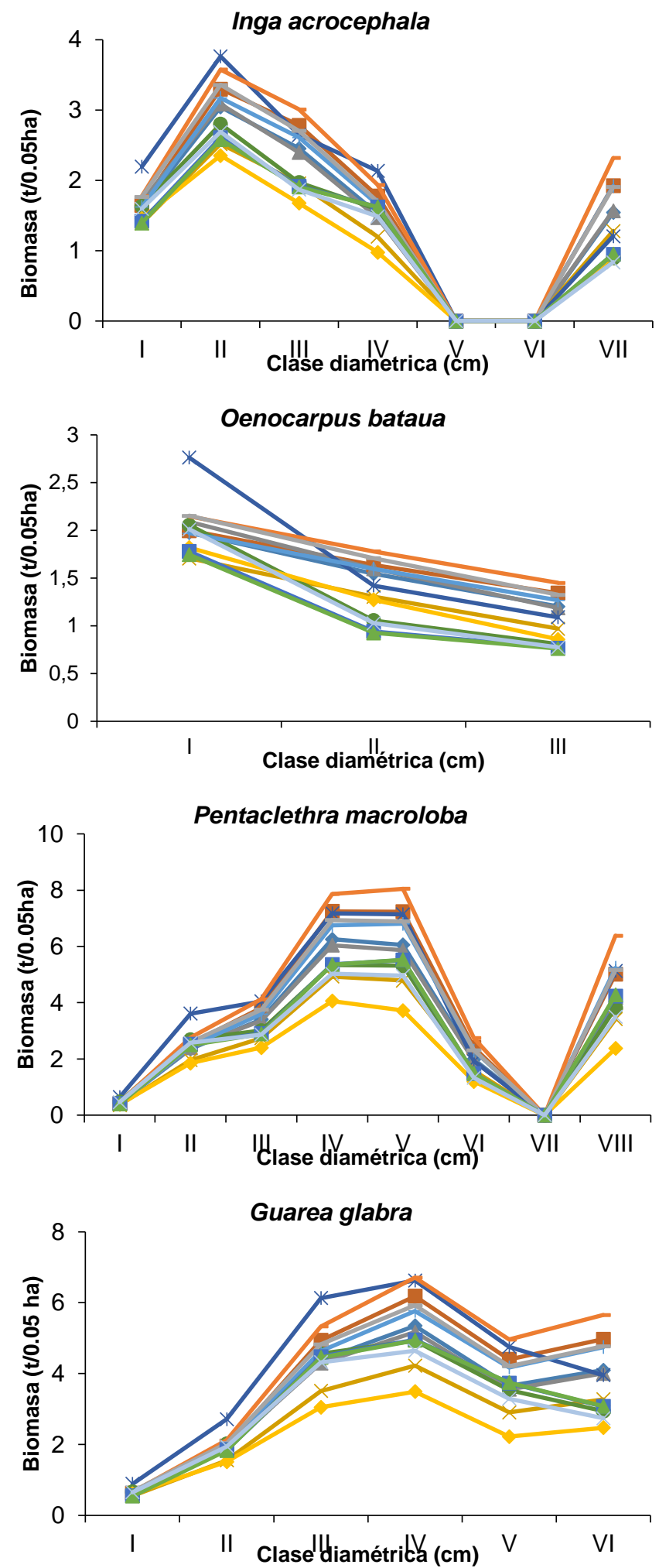

Figura 1b. Distribución de biomasa aérea por clase diamétrica para especies y zonobioma según modelos alométricos $(2 / 3)$ 


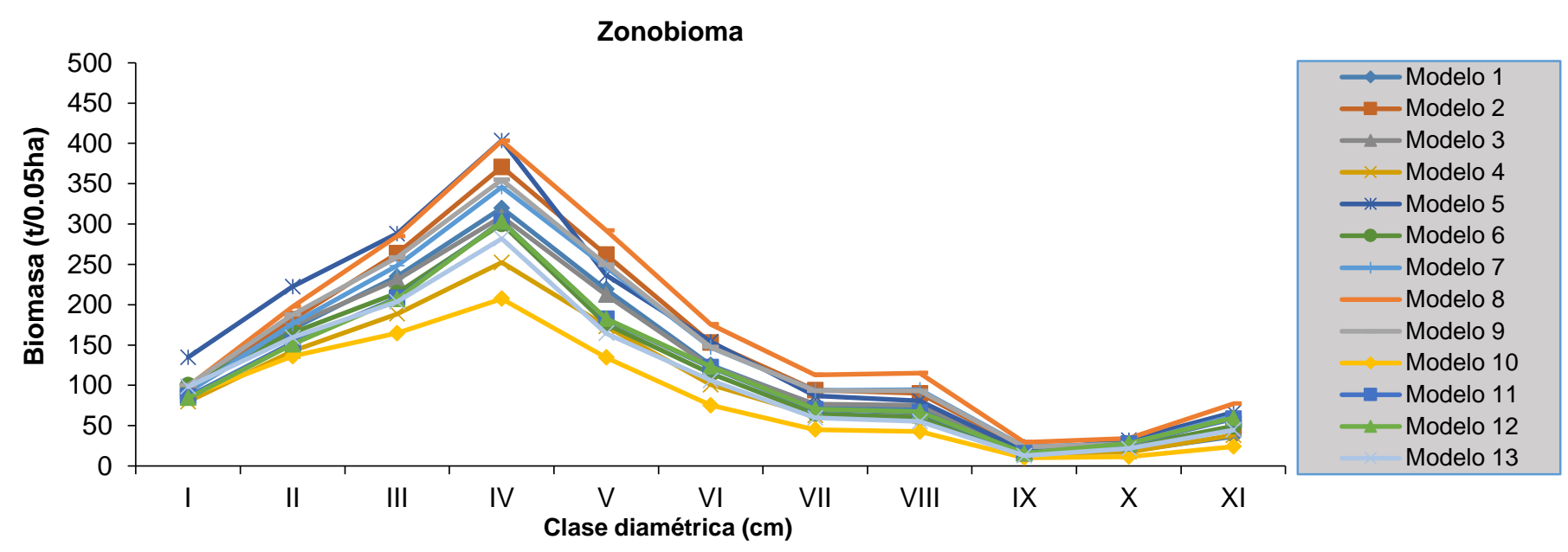

Figura 1c. Distribución de biomasa aérea por clase diamétrica para especies y zonobioma según modelos alométricos

Se encuentra que los modelos 8,2 y 5 presentan las estimaciones más altas, los dos primeros estiman la biomasa en función del diámetro a la altura del pecho, el modelo 5 lo hace en función de la variable altura total. La menor estimación para todas las especies (exceptuando la palma) y para todas las clases diamétricas se obtiene con el modelo 10 que incorpora únicamente diámetro a la altura del pecho, seguido del modelo 4 y 13 el cual tiene en cuenta la variable altura total.
Los modelos presentan entre sí un comportamiento afín en la especie Eschweilera coriacea a diferencia de lo que muestran las gráficas de las especies Dacryodes colombiana e Iryanthera juruensis. El modelo 5 es el que expresa el comportamiento más atípico en todos los casos.

La distribución de la biomasa expresada en kg por hectárea para todo el zonobioma, por diámetro según los trece modelos alométricos se evidencia en la Figura 2.

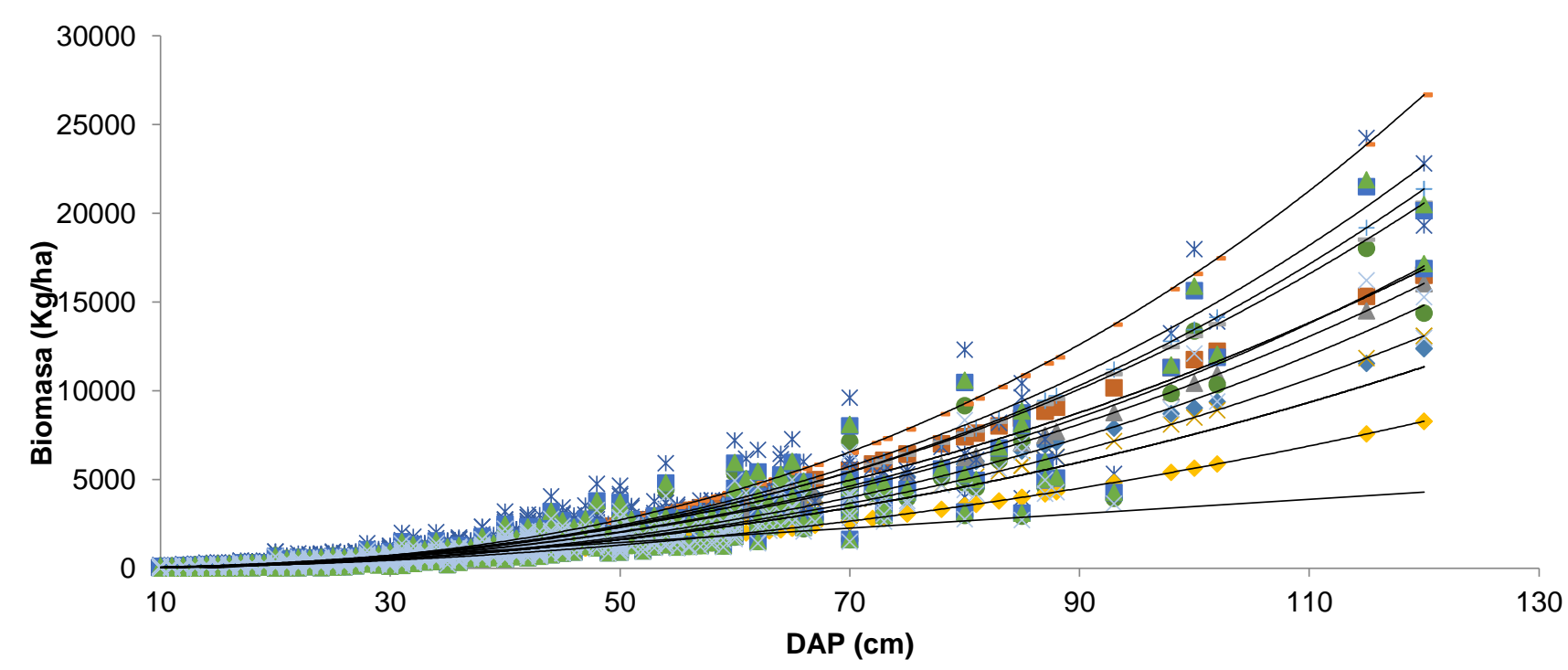

Figura 2. Biomasa estimada para zonobioma por diámetro según modelos alométricos

Para el zonobioma húmedo tropical la mayor estimación de biomasa la reporta el modelo 8 y la menor, el modelo 10. Comparando las estimaciones en kilogramos por hectárea por diámetro arrojadas por todos los modelos para el zonobioma, se aprecia que en los diámetros inferiores los valores son más similares a diferencia de los diámetros superiores por encima de 70 $\mathrm{cm}$ donde se evidencia más disparidad entre los valores de biomasa, registrando un mayor error.

\subsection{Variables estructurales y biomasa}

Se resumen en la Tabla 4 el valor medio de las variables estructurales mostrando los rangos en los que se mueve el valor, y carbono almacenado expresado en toneladas; seguido, se enuncian los resultados de las pruebas de varianza (Kruskal-Wallis). 
Tabla 4. Valores medios de variables estructurales, biomasa y carbono comparados mediante prueba no paramétrica Kruskal-Wallis

\begin{tabular}{|c|c|c|c|c|c|}
\hline Especie & Altura total $(\mathrm{m})$ & $\begin{array}{l}\text { Abundancia } \\
\text { (Ind/0.05ha) }\end{array}$ & $\begin{array}{l}\text { Área } \\
\left(\mathrm{m}^{2} / 0.05 \mathrm{ha}\right)\end{array}$ & $\begin{array}{r}\text { basalBiomasa } \\
(\mathrm{t} / 0.05 \mathrm{ha})\end{array}$ & $\begin{array}{l}\text { Carbono } \\
\text { (t/0.05ha) }\end{array}$ \\
\hline Micropholis guyanensis & 15.8 & 24.2 & 1.77 & $26.13 \pm 0.27$ & 13.06 \\
\hline Dacryodes colombiana & 14.8 & 23.8 & 0.83 & $11.98 \pm 0.15$ & 5.99 \\
\hline Abarema jupunba & 15.0 & 23.3 & 1.00 & $14.78 \pm 0.25$ & 7.39 \\
\hline Iryanthera juruensis & 16.4 & 22.5 & 1.05 & $15.40 \pm 0.25$ & 7.70 \\
\hline Inga acrocephala & 15.1 & 23.5 & 0.79 & $11.43 \pm 0.22$ & 5.71 \\
\hline Guarea glabra & 17.9 & 26.5 & 1.53 & $22.43 \pm 0.55$ & 11.21 \\
\hline Zonobioma húmedo tropical & 16.25 & 92.84 & 4.59 & $78.49 \pm 0.39$ & 39.24 \\
\hline Estadístico H $(10, \mathrm{~N}=855)$ & 81.33 & 438.8 & 117.26 & 188.57 & \\
\hline Nivel de significancia $(p)$ & $8.768 \mathrm{e}-16$ & $<2.2 \mathrm{e}-16$ & $<2.2 \mathrm{e}-16$ & $<2.2 \mathrm{e}-16$ & \\
\hline
\end{tabular}

La biomasa estimada varían entre $48.17 \pm 0.64 \mathrm{t} / 0.05$ para Aspidosperma sp. y $5.18 \pm 0.07 \mathrm{t} / 0.05$ ha para Oenocarpus bataua, por su parte el carbono almacenado oscila entre $24 \mathrm{t} / 0.05$ y 2,6 t/0.05 para las mismas especies. Inga acrocephala y Dacryodes colombiana mostraron valores bajos por debajo del promedio con relación a las otras especies.

Los valores extremos de altura total corresponden a las especies Aspidosperma sp y $O$. bataua (20.4 m y $12.2 \mathrm{~m}$ respectivamente); el número de individuos varía de 26 a 22 en Guarea glabra y Iryanthera juruensis y el área basal presenta su valor máximo en Aspidosperma sp $\left(3.05 \mathrm{~m}^{2}\right)$ y su mínimo en Pentaclethra macroloba $\left(1.88 \mathrm{~m}^{2}\right)$.

La biomasa promedio para el zonobioma húmedo tropical es de $78.49 \pm 0.39 \mathrm{t} / 0.05 \mathrm{ha}$ estando muy por encima de lo que reportan las diez especies. La altura total promedio es de $16.25 \mathrm{~m}$, por debajo de la media de las especies Aspidosperma sp. Eschweilera coriacea, Iryanthera juruensis, Pentaclethra macroloba y Guarea glabra. El número de individuos en 0.05 ha es de alrededor de 92 y el área basal media es de 4.59 $\mathrm{m}^{2} / 0.05 \mathrm{ha}$, valor superado únicamente por el que reporta Aspidosperma sp. El zonobioma presenta 39.24 toneladas de carbono/0.05 ha aproximadamente.
Las pruebas de varianza (Kruskal-Wallis) determinaron que todas las variables presentan diferencias significativas (probabilidad de $0.05 \%$ ), lo que indica que la estructura y composición y el grado de disturbio de la especie, están relacionados con la cantidad de biomasa almacenada en la vegetación, según lo expresado en [2].

Los diagramas de caja tipo Box-Plot de la Figura 3 visualizan la existencia de valores atípicos propios de formaciones vegetales tropicales húmedas. Además, la distribución y simetría de los datos de altura total y área basal; esta situación es muy frecuente en este tipo de bosques sujetos a perturbaciones de baja intensidad y media frecuencia, reafirmando la configuración de un mosaico sucesional altamente diverso.

En la Tabla 5 los valores de altura total corresponden a los máximos y mínimos expresados en metros; los valores de abundancia, área basal, biomasa y carbono corresponden al promedio de cada especie. El total de altura hace referencia al valor mínimo y máximo de todos los individuos muestreados para esa especie, el total de abundancia, área basal, biomasa y carbono es la suma del total de valores medios de todas las clases diamétricas de esa especie.
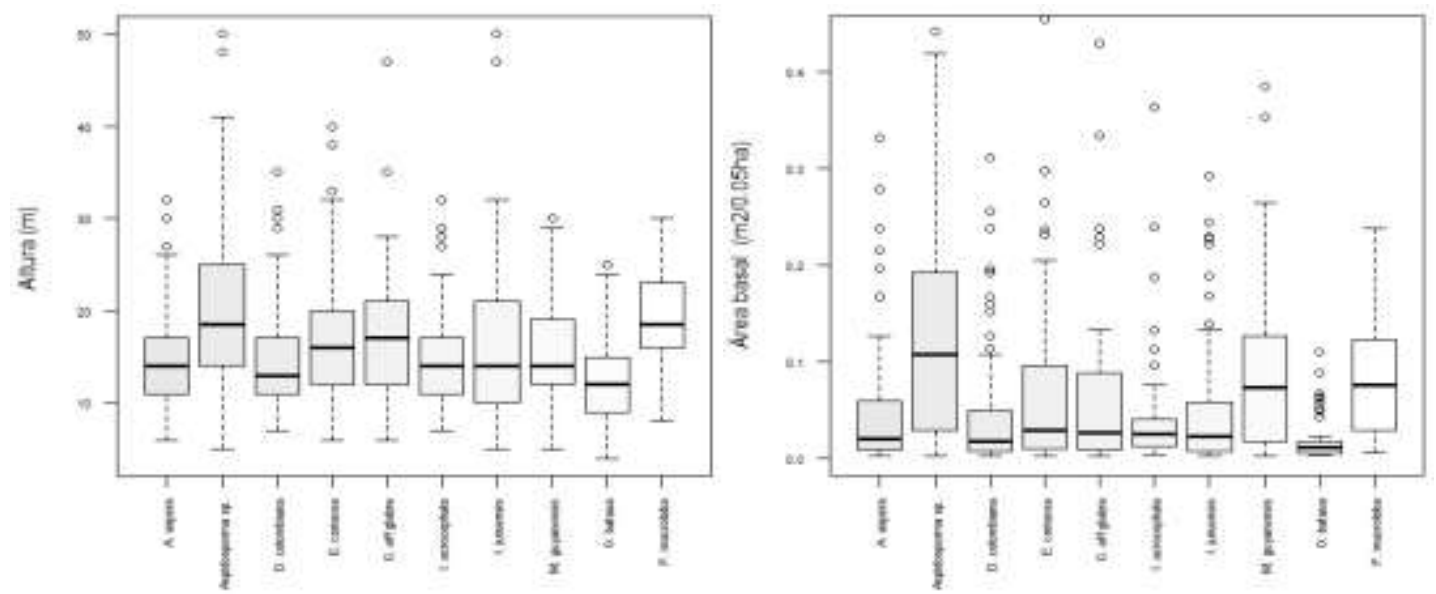

Figura 3. Diagramas de caja Box-Plot para altura total y área basal 
Tabla 5. Distribución de variables de la estructura de la vegetación, biomasa y carbono por clase diamétrica (1/2)

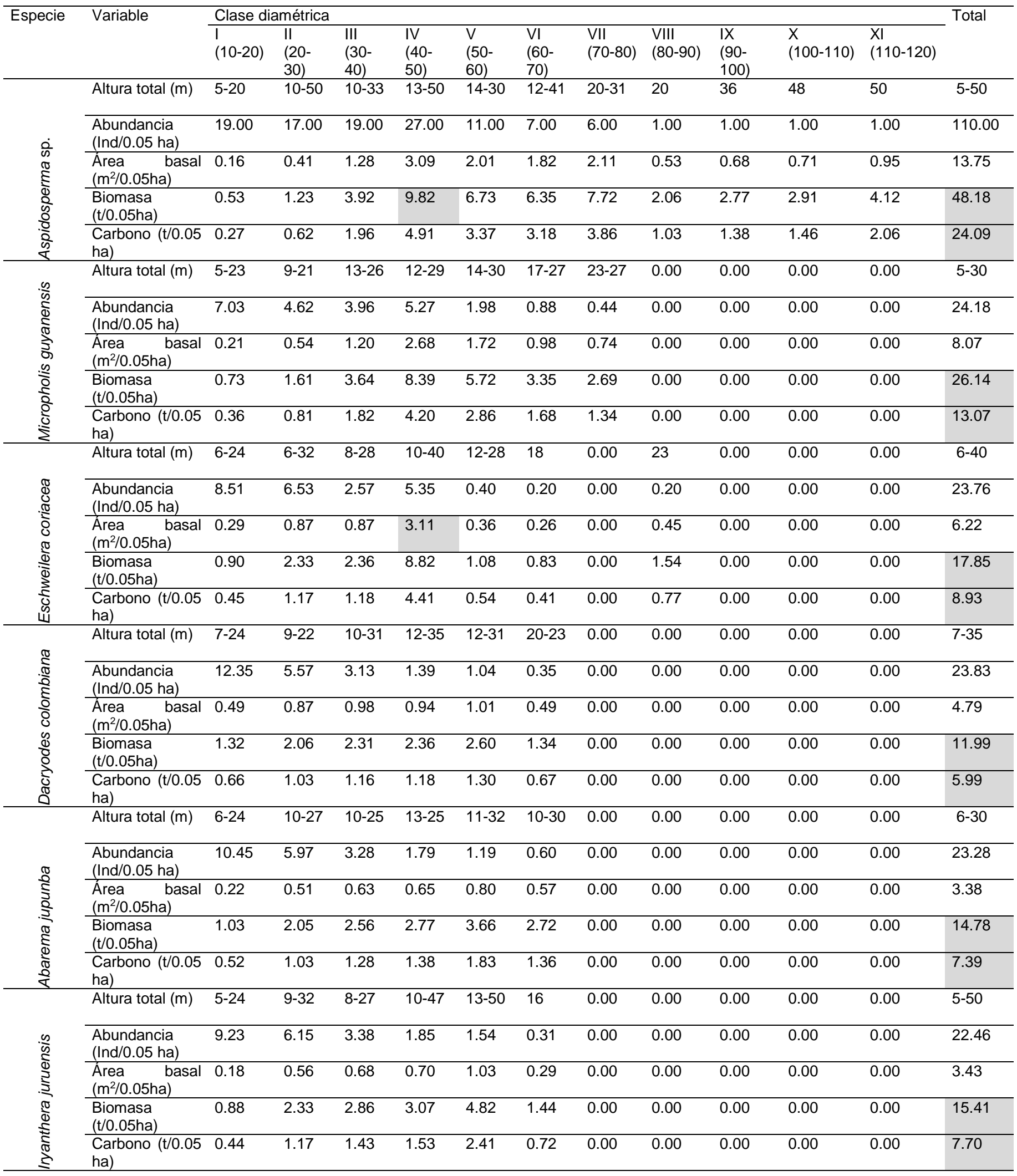


Tabla 5. Distribución de variables de la estructura de la vegetación, biomasa y carbono por clase diamétrica (2/2)

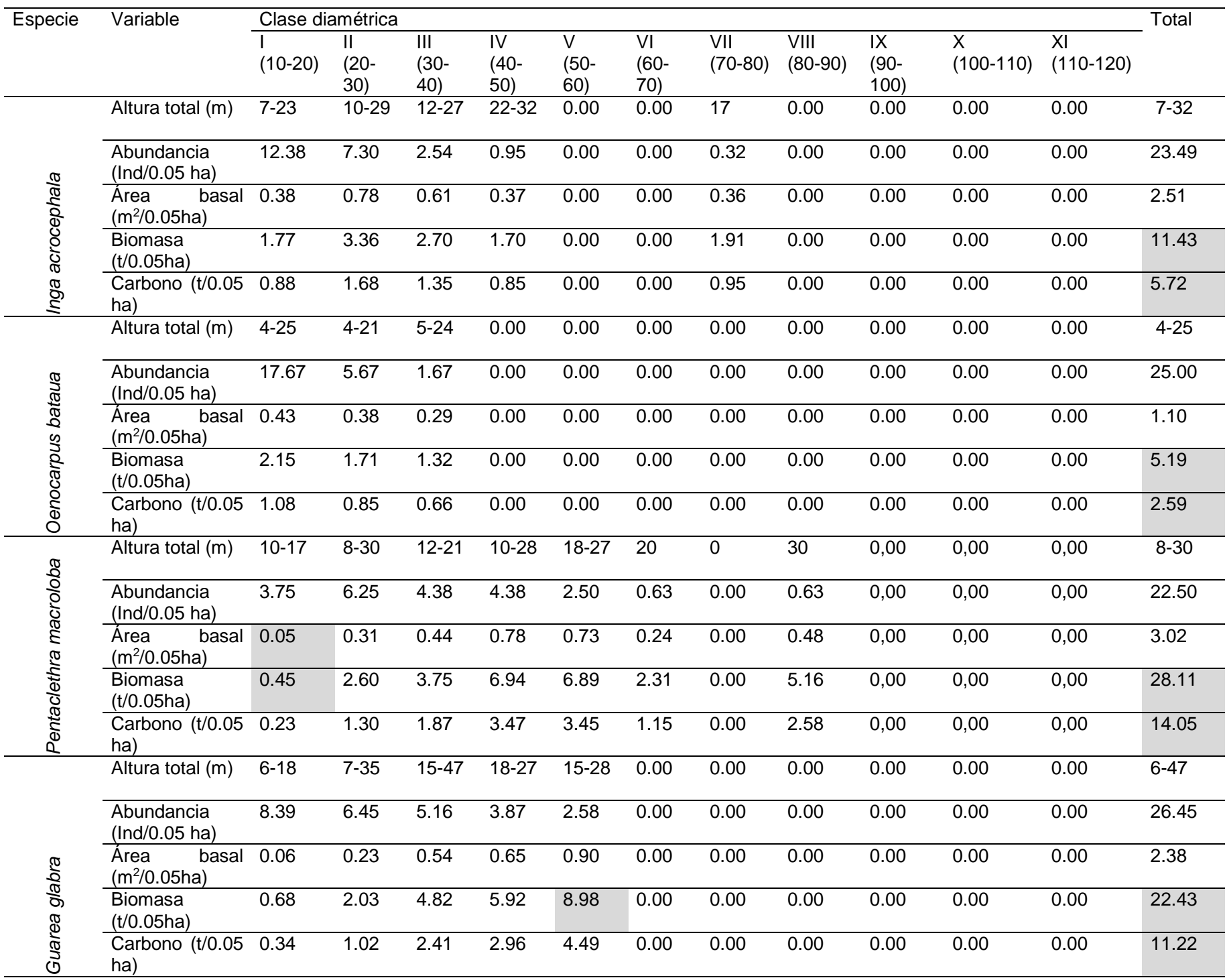

La distribución de variables por cada clase diamétrica muestra evidencia que la altura total para las diez especies oscila entre $4 \mathrm{~m}$ y $50 \mathrm{~m}$; la abundancia registra la mayor cantidad de individuos en la primera clase para todas las especies a excepción de la más representativa Aspidosperma sp. que los registra en las clases IV y III y contiene el valor más alto con 27 individuos $/ 0.05 \mathrm{ha}$, para el resto de las especies existe la tendencia de que en las clases diamétricas mayores (>90 cm DAP) no haya presencia de individuos. El mayor valor de área basal lo tiene la especie Eschweilera coriacea (3.11 m²/0.05ha) en la clase diamétrica IV, el menor es para Pentaclethra macroloba (0.05 m²/0.05ha) en la clase I.

En cuanto a biomasa los valores más altos son de $9.82 \mathrm{t} / 0.05 \mathrm{ha}$ y $8.98 \mathrm{t} / 0.05 \mathrm{ha}$ en las especies Aspidosperma sp. y Guarea glabra; la especie Pentaclethra macroloba con 0.45 t/0.05ha en la clase diamétrica I es la que menos biomasa almacena. Las clases diamétricas donde más carbono se almacena son la IV $(40-50 \mathrm{~cm})$ y V $(50-60 \mathrm{~cm})$

\subsection{Distribución de biomasa y carbono por clase diamétrica}

En la Figura 4 se relacionan para cada especie sus valores de biomasa y carbono expresados en toneladas por cada 0.05 ha para cada clase diamétrica.

La mayor cantidad de biomasa y carbono se reporta en las clases diamétricas IV y V , con una disminución en las clases inferiores y una evidente variabilidad entre especies; esta condición en el almacenamiento de carbono en el bosque estudiado posiblemente se debe a la acumulación de número de individuos por unidad de área en esas categorías diamétricas, resultado que refuerza el principio silvicultural de la opción de cosechar las acumulaciones de biomasa de clases emergentes y sobremaduras, en beneficio de la ampliación de la capacidad de acumulación de carbono de estos bosques. También responde a lo propuesto en $[4,7]$, donde se menciona que la cantidad de carbono almacenado es los ecosistemas forestales es muy variable y su capacidad de almacenar varía en función 
de la composición florística, la abundancia de la población y el estado de desarrollo del mismo.

Además, el uso de ecuaciones alométricas se ve condicionado por factores como el volumen, la densidad básica y el coeficiente de expansión del árbol, siendo la variable que mayor influencia tiene en los resultados de biomasa [17].

\section{Aspidosperma sp.}

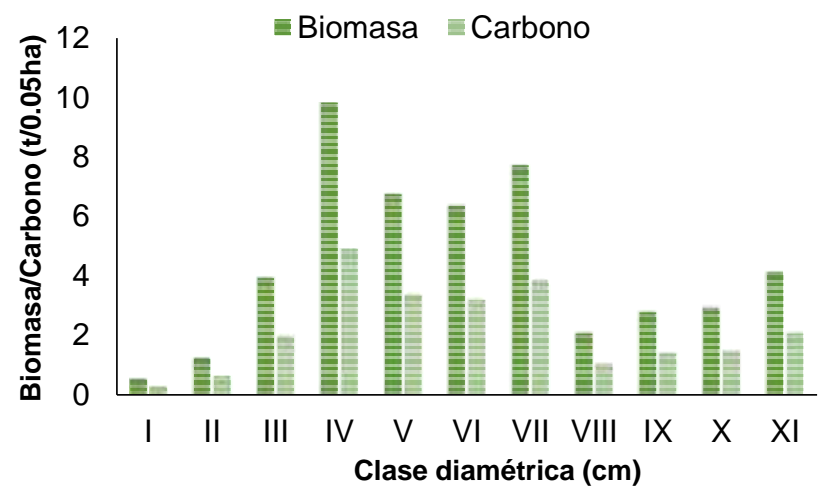

Eschweilera coriacea

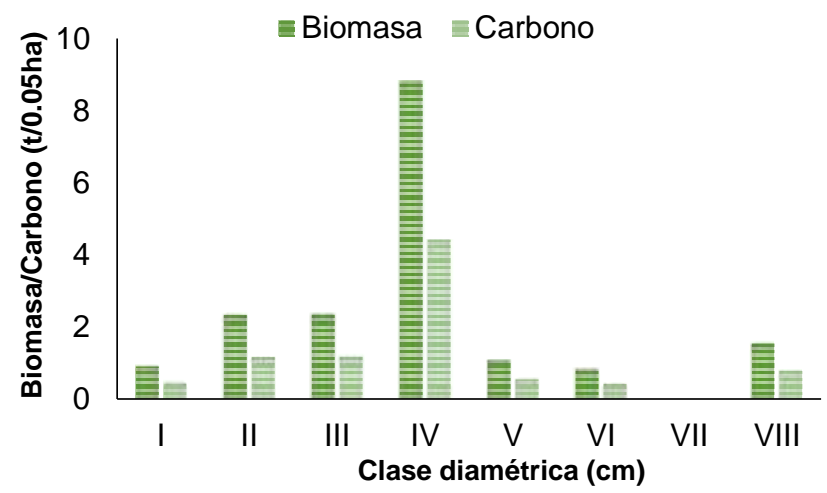

Abarema jupunba

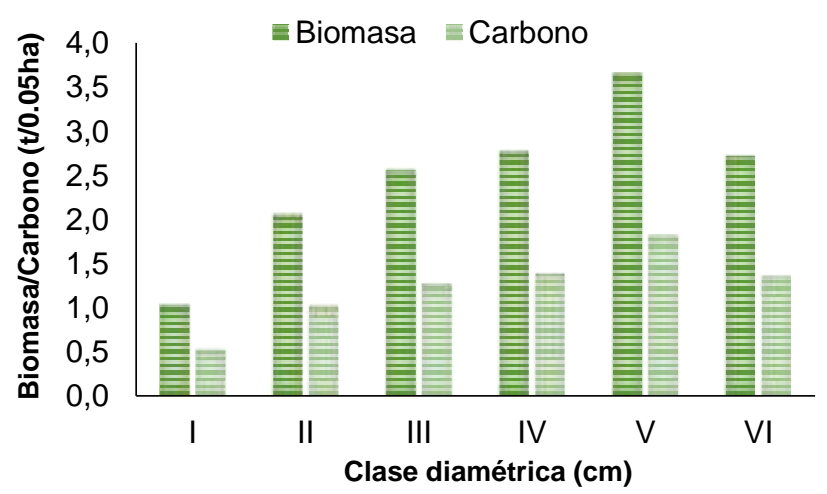

Algunos estudios en Latinoamérica sugieren el desarrollo de funciones específicas para las variables dasométricas en cuestión (por ejemplo, altura total) que permitan una mayor precisión en las estimaciones y aprovechando mejor los datos obtenidos en los inventarios [18].
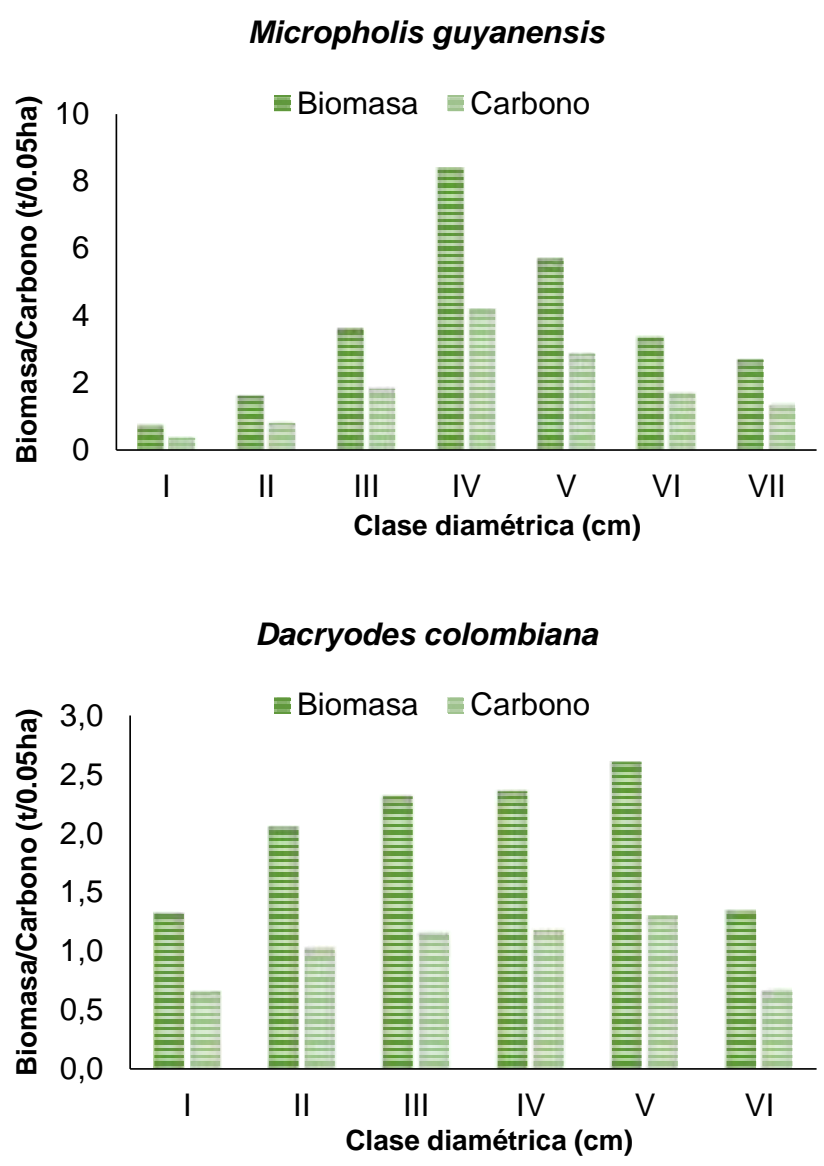

Figura 4. Distribución de biomasa aérea y carbono almacenado por clase diamétrica (1/2)

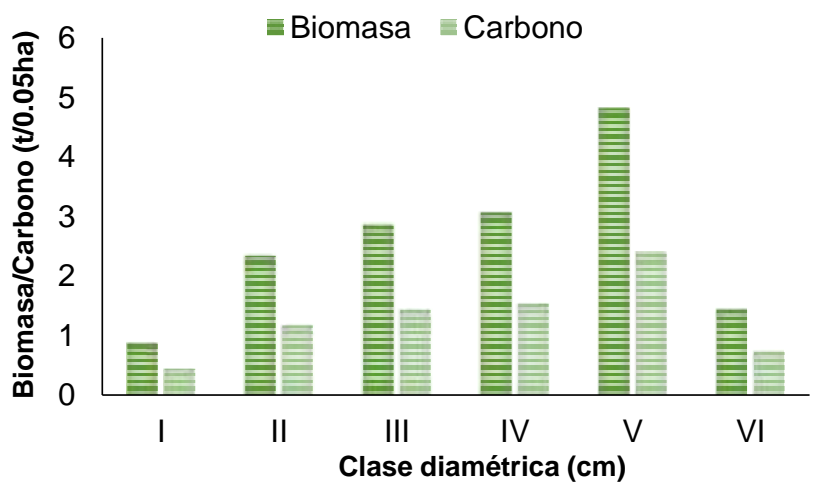




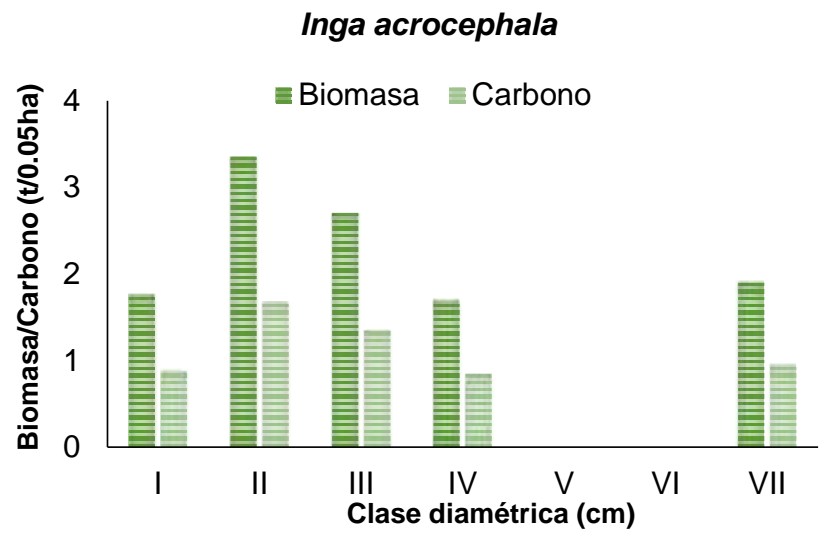

Pentaclethra macroloba

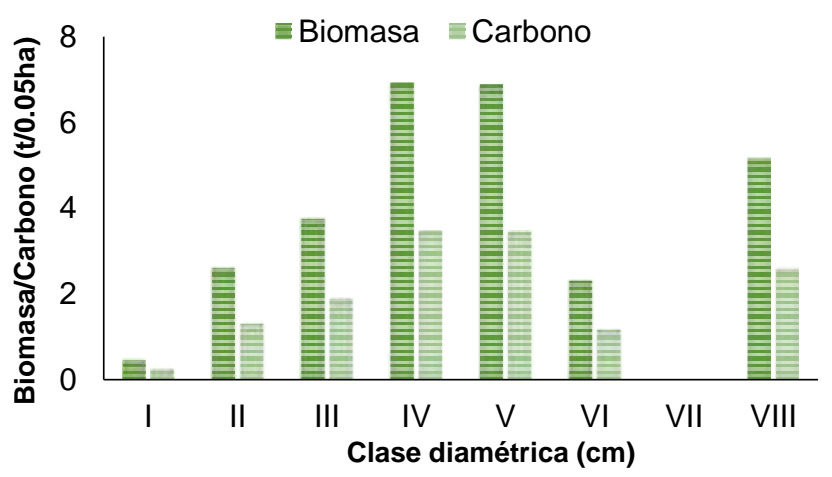

Oenocarpus bataua

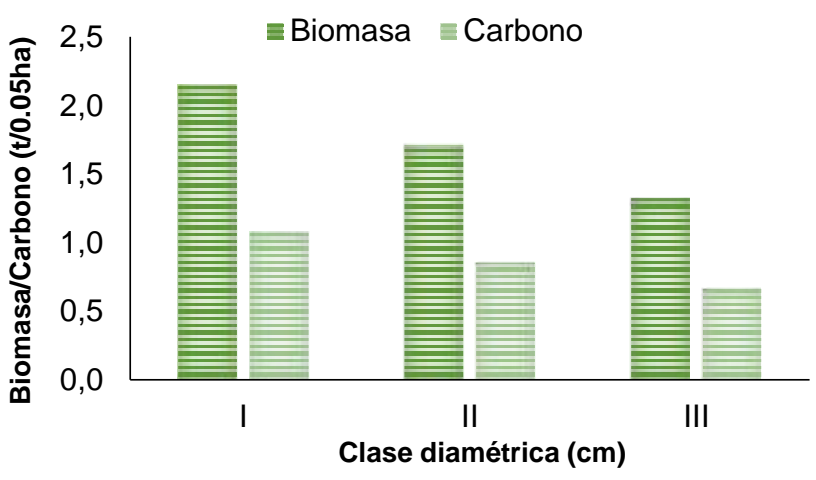

Guarea glabra

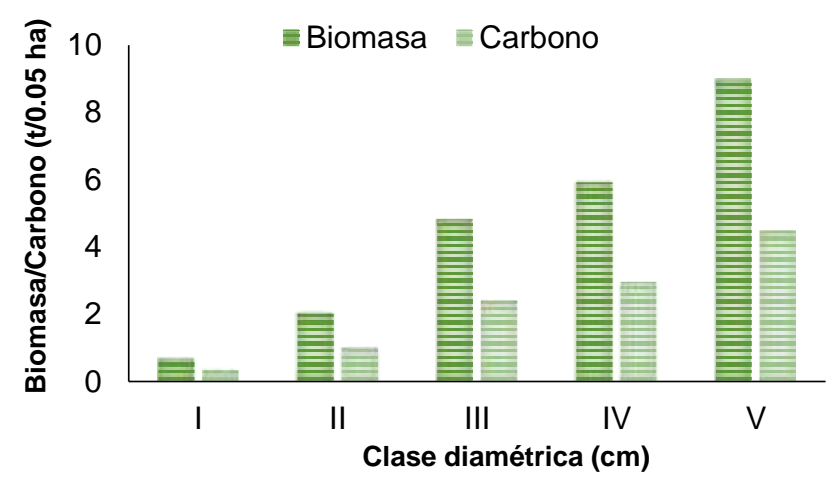

Figura 4. Distribución de biomasa aérea y carbono almacenado por clase diamétrica (2/2)

\section{Conclusiones}

Los modelos basados únicamente en la variable diámetro a la altura del pecho muestran supremacía en el ajuste, es necesario considerar algunos aspectos propios de la silvicultura, debido a que los programas nacionales y regionales de combate contra la deforestación constantemente buscan obtener valores más altos de biomasa. La incorporación a estos modelos de otras variables puede hacer más compleja su manipulación matemática y un esfuerzo adicional en la ampliación de la muestra en campo. Sin embargo, se mejora el análisis, especialmente asociado con la cuantificación de la degradación, dinámica y potencial de adaptación a la variabilidad climática.

Al observar los valores de biomasa arrojados por todos los modelos alométricos para las clases inferiores y superiores, se encuentra que el error de estimación es mayor en las clases superiores debido a que los modelos de este tipo tienden a sobre estimar los árboles de gran porte. La poca presencia de individuos con diámetros mayores a $80 \mathrm{~cm}$, disminuyen proporcionalmente la cantidad de biomasa en el ecosistema y por consiguiente las reservas de carbono. La anterior situación hace necesario evaluar los factores externos que ejercen presión sobre estos individuos y buscar estrategias que garanticen su estadio en el bosque.
Valores de biomasa de 130,44 t/ha y $178,94 \mathrm{t} /$ ha se presentan en $[19,20]$ para bosques húmedos tropicales, muy por debajo de lo encontrado con la presente metodología; esta situación puede justificarse porque en estas investigaciones se tienen en cuenta variables como la hojarasca producida, calidad del fuste, forma de la copa en el primer caso y densidad de la madera en el segundo, lo que arroja resultados con menor error y cercanos a la realidad del bosque.

Para finalizar, se recomienda utilizar modelos alométricos que incorporen otras variables además del diámetro a la altura del pecho, como altura comercial, densidades de la madera y estructura de copa, entre otras, porque suelen ser las responsables de un alto porcentaje de la variación en la biomasa, evitando sobreestimaciones y mostrando una situación más concreta del comportamiento del bosque estudiado.

\section{Agradecimientos}

Los autores reconocen el apoyo de la Universidad Distrital Francisco José de Caldas, de los semilleros de investigación Productos y Procesos del Bosque (PROPROBOS) y Producción y Manejo Forestal (PROMAFOR); y la contribución de la Ingeniera Forestal L. M. Montenegro en el aporte de los datos de campo, en el marco del Plan de Ordenación Forestal del Municipio de Río Quito. 


\section{Referencias}

[1] J. A. Benjamín Ordóñez and O. Masera, "Captura de carbono ante el cambio climático," Madera y Bosques, vol. 7, no. 1, pp. 312, 2001.

[2] J. O. Rangel Churio, Colombia Diversidad Biótica XII: La región Caribe de Colombia, 1st ed. Bogotá, Colombia: Universidad Nacional de Colombia, 2012, ch.: Estructura, biomasa aérea y carbono almacenado en los bosques del sur y noroccidente de Córdoba, pp. 923-961.

[3] E. D. Schulze, C. Wirth, and M. Heimann, "Managing forests after Kyoto," Science, vol. 289, no. 5487, pp. 2058-2059, 2000.

[4] M. Acosta Mireles, J. Vargas Hernández, A. Velázquez Martínez, and J. Etchevers Barra, "Estimación de la biomasa aérea mediante el uso de relaciones alométricas en seis especies arbóreas en Oaxaca, México," Agrociencia, vol. 36, no. 6, pp. 725-736, 2002.

[5] R. Dixon, "Agroforestry systems: sources of sinks of greenhouse gases?," Agroforestry Systems, vol. 31, no. 2, pp. 99-116, 1995.

[6] T. Lapeyre, J. Alegre, and L. Arévalo, "Determinación de las reservas de carbono de la biomasa aérea, en diferentes sistemas de uso de la tierra en San Martín, Perú," Ecología Aplicada, vol. 3, no. 1, pp. 35-44, 2004.

[7] B. Schlegel, "Estimación de la biomasa y carbono en bosques del tipo forestal siempreverde," in Symp. Internacional Medición y Monitoreo de la Captura de Carbono en Ecosistemas Forestales '01, Valdivia, Chile, 2001, pp. 1-13.

[8] G. Galindo García, E. Cabrera Montenegro, D. M. Vargas Galvis, H. R. Pabón Méndez, K. R. Cabrera Torres, A. P. Yepes Quintero, J. F. Phillips Bernal, D. A. Navarrete Encinales, A. J. Duque Montoya, M. C. García Dávila, and M. F. Ordóñez Castro, "Estimación de la biomasa aérea usando datos de campo e información de sensores remotos," Instituto de Hidrología, Meteorología, y Estudios Ambientales (IDEAM), Bogotá, Colombia, Nov. 2011.

[9] R. Moreno and M. X. González. (2011) Centro de Estudios para la Justicia Social Tierra Digna Homepage. Accessed jul 2017. [Online]. Available: http://tierradigna.net/attachments/article/17/Accio\%CC\%81n\%2 OPopular\%20Ri\%CC\%810\%20Quito\%20(Def).pdf

[10] IFCAYA Ltda., "Plan de ordenación forestal para una zona de 74400 ha en Bosque muy húmedo tropical de la región de Napipí, Choco," Instituto Nacional de los Recursos Naturales Renovables y del Ambiente (INDERENA), Quibdó, Colombia. 1975.
[11] P. Leyva, Riqueza de especies y composición florística de las comunidades de plantas de la Región del Chocó: una actualización. Bogotá, Colombia: Fondo para la Protección del Medio Ambiente, 1993, ch.: Colombia Pacífico, pp. 200-219.

[12] J. O. Rangel Churio, P. Lowy, and M. Aguilar, Colombia diversidad biótica II. Tipos de vegetación en Colombia. Bogotá, Colombia: Instituto de Ciencias Naturales Universidad Nacional de Colombia, 1997, ch.: Métodos de estudio de la vegetación, pp. 59-87.

[13] A. M. Campo and V. S. Duval, "Diversidad y valor de importancia para la conservación de la vegetación natural," Anales de Geografía, vol. 34, no. 2, pp. 25-42, 2014.

[14] A. Novoa Leyva, J. Santoyo Rodríguez, E. Hernández Álvarez, D. Pelz, C. Rodríguez Franco, A. Gallegos Rodríguez, and M. G. Lomelí Ramírez, "El Î́ndice de Valor de Importancia de especies forestales en base a unidades ecológicas de un bosque tropical," in Conv. Semana de la Investigación Científica '05, Ciudad de México, México, 2005, pp. 122-128.

[15] V. Teixeira Roth and E. Sánchez Infantas, "Patrones poblacionales de las principales especies herbáceas en la Reserva Nacional de Lachay," Ecología Aplicada, vol 5, no. 1, pp. 23-27, 2006.

[16] Intergovernmental Panel on Climate Change, "Special report on renewable energy sources and climate change mitigation," Intergovernmental Panel on Climate Change (IPCC), New York, USA, Jun. 2011.

[17] J. Burgos and J. A. Pérez Rojas, "Modelos de regresión no lineal para la estimación de biomasa en algunos ecosistemas boscosos de Colombia," Colombia Forestal, vol. 8, no. 17, pp. 103-109, 2004.

[18] E. Dauber, J. Terán, and R. Guzmán, "Estimaciones de biomasa y carbono en bosques naturales de Bolivia," Revista Forestal Iberoamericana, vol. 1, no. 1, pp. 13-23, 2000.

[19] N. J. Quiceno and G. M. Tangarife, "Estimación del contenido de biomasa, fijación de carbono y servicios ambientales, en un área de bosque primario en el resguardo indígena Piapoco Chiguirochatare de Barrancominas, departamento del Guainía," Master's thesis, Universidad de Manizales, Manizales, Colombia, 2013.

[20] H. Quinto Mosquera and F. Moreno Hurtado, "Dinámica de la biomasa aérea en un bosque pluvial tropical del Chocó Biogeográfico," Revista Facultad Nacional de Agronomía, vol. 64, no. 1, pp. 5917-5936, 2011. 